\title{
INFERÊNCIA BAYESIANA NA AVALIAÇÃO GENÉTICA DE BOVINOS DA RAÇA TABAPŨ̃ DO NORDESTE BRASILEIRO ${ }^{1}$
}

\author{
ARACELE PRATES DE OLIVEIRA ${ }^{2 *}$, CARLOS HENRIQUE MENDES MALHADO ${ }^{3}$, \\ LEANDRO TEIXEIRA BARBOSA ${ }^{4}$, RAIMUNDO MARTINS FILHO ${ }^{5}$, PAULO LUIZ SOUZA CARNEIRO ${ }^{6}$
}

\begin{abstract}
RESUMO - Objetivou-se comparar por meio do Fator de Bayes (FB), modelos para estimar componentes de covariância, parâmetros genéticos e valores genéticos para os pesos ajustados aos 205 (P205), 365 (P365) e 550 (P550) dias de idade em animais da raça Tabapuã, criados a pasto no Nordeste do Brasil. Foram utilizadas informações de 26.838 animais no período entre 1975 a 2007 provenientes do controle de desenvolvimento ponderal da Associação Brasileira de Criadores de Zebu (ABCZ). Os componentes de covariância e parâmetros genéticos foram estimados pela abordagem bayesiana em análises uni e bicaracterísticas utilizando-se o programa GIBBS3F90. O modelo completo proporcionou melhor ajuste para a estimativa dos parâmetros genéticos para as características P205 e P365. Quanto à característica P550, o modelo que proporcionou o melhor ajuste foi o que não incluiu o efeito de ambiente permanente. As tendências genéticas de efeito direto foram significativas para as características P205, P365 e P550, correspondendo a incrementos de 2,67 kg, 7,35 kg e 8,32 kg, respectivamente, durante os 32 anos avaliados. As tendências fenotípicas foram significativas apenas para as características P205 e P365, correspondendo a incrementos de 26,82 kg e 22,42 kg, respectivamente. As correlações genéticas entre o peso aos 205, 365 e 550 dias de idade foram de alta magnitude (P205P365=0,90; $\mathrm{P} 205 \mathrm{P} 550=0,90$ e P365P550=0,98), podendo-se obter ganhos genéticos via resposta correlacionada para os pesos aos 365 e 550 dias de idade ao selecionar o P205.
\end{abstract}

Palavras-chave: Correlação genética. Ganho genético. Herdabilidade. Tendência genética. Zebu.

\section{BAYESIAN INFERENCE IN THE GENETIC EVALUATION OF THE BRAZILIAN NORTHEAST TABAPUÃ CATTLE}

\begin{abstract}
The objective was to compare models by Bayes factor (BF) to estimate the (co)variance components, genetic parameters and genetic values for the adjusted weights at 205 (W205), 365 (W365) and 550 (W550) days of age in Tabapuã animals reared on pasture in the Brazilian Northeast. Information about 26,838 cattle born in the period from 1975 to 2007 originally from the weight-development-control system of the Brazilian Association of Zebu Farmers (Associação Brasileira de Criadores de Zebu, ABCZ) was used. The (co) variance components and genetic parameters were estimated by the Bayesian approach, in single- and two-trait analysis, using the GIBBS3F90 software. The full model provided the best fit to estimate the breeding value for W205 and W365 characteristics parameters. For trait W550, the model that provided the best fit was the one that did not include the effect of permanent environment. Genetic trends of direct effect were significant for the W205, W365 and W550 features, corresponding to increments of $2.67 \mathrm{~kg}, 7.35 \mathrm{~kg}$ and $8.32 \mathrm{~kg}$, respectively, during the 32 years evaluated. Phenotypic trends were significant only for W205 and W365 characteristics corresponding to increments of $26.82 \mathrm{~kg}$ and $22.42 \mathrm{~kg}$, respectively. The genetic correlations between weight at 205, 365 and 550 days of age were of high magnitude $(\mathrm{P} 205 \mathrm{P} 365=0.90,=0.90$ and P205P550 P365P550 $=$ 0.98 ); you can obtain genetic gains via correlated response to the weights at 365 and 550 days old when selecting for W205.
\end{abstract}

Keywords: Genetic correlation. Genetic gain. Heritability. Genetic tendency. Zebu.

\footnotetext{
*Autor para correspondência

${ }^{1}$ Recebido para publicação em 09/08/2014; aceito em 31/08/2015

Tese de Doutorado em Zootecnia do primeiro autor.

${ }^{2}$ Pós Doutoranda, Pesquisadora Fapepi/CNPq, Corrente (PI), Brasil; araceleprates@hotmail.com.

${ }^{3}$ Departamento de Ciências Biológicas, Universidade Estadual do Sudoeste da Bahia, Jequié (BA), Brasil.

${ }^{4}$ Departamento de Zootecnia, Universidade Federal do Sergipe, São Cristóvão (SE), Brasil.

${ }^{5}$ Universidade Federal do Ceará, Cariri (CE), Brasil.

${ }^{6}$ Departamento de Ciências Biológicas, Universidade Estadual do Sudoeste da Bahia, Jequié (BA), Brasil.
} 


\section{INTRODUÇ̃̃O}

Os bovinos Tabapuã constituem uma raça brasileira originada de cruzamentos entre o gado mocho nacional e animais de origem indiana. Foi na década de 40, no município de Tabapuã, estado de São Paulo, que a raça assumiu algumas das características que perduram até hoje. Sua história tem início em 1907 na região de Leopoldo de Bulhões, estado de Goiás. Em 1970, o Ministério da Agricultura recomendou que o Tabapuã fosse incluído entre as raças zebuínas, ainda como "tipo". A, sendo a Associação Brasileira de Criadores de Zebu (ABCZ) encarregada de realizar o registro genealógico da raça. Em 1981, foi definitivamente reconhecido como raça, sendo o terceiro neozebuíno a ser formado no mundo, depois do Brahma e do Indubrasil (ABCT, 2014).

A seleção é o principal método, dentro de um programa de melhoramento genético, utilizado para escolher os melhores animais para a reprodução. A avaliação genética depende de estimativas de parâmetros genéticos acuradas para as características de crescimento. A acurácia da estimativa desses parâmetros depende, principalmente, do número de informações utilizadas na análise, do modelo estatístico escolhido e do método de estimação dos componentes de covariância e parâmetros genéticos das características estudadas (BARBOSA et al. 2008).

Estimativas acuradas dos componentes de variância têm sido importantes na produção animal, uma vez que as diferenças entre o valor verdadeiro e o valor estimado aumentam o erro de predição dos valores genéticos. No entanto, para a obtenção dessas estimativas é essencial identificar um método estatístico adequado e que melhor represente o comportamento biológico das características em estudo. O método da Máxima Verossimilhança Restrita (REML) é o mais utilizado para estimar componentes de covariância e parâmetros. Todavia, métodos bayesianos têm propiciado novas perspectivas no que toca a questões relacionadas à estimação de componentes de variância e parâmetros genéticos.

A inferência bayesiana combina a informação a priori e a função de verossimilhança. Esta combinação é feita por meio do Teorema de Bayes, originando a distribuição a posteriori. Contudo, a maioria dos trabalhos referentes à inferência bayesiana, no Brasil, aborda somente os componentes de covariância e parâmetros genéticos, não considerando os valores genéticos para a estimação dos ganhos genéticos por meio das tendências. Nessa ótica, o objetivo deste estudo foi estimar parâmetros genéticos, tendências genéticas, maternas e fenotípicas e identificar o melhor ajuste de modelo em características produtivas de animais da raça Tabapuã criados no Nordeste do Brasil.

\section{MATERIAL E MÉTODOS}

Os dados utilizados foram provenientes do controle de desenvolvimento ponderal da raça Tabapuã, da Associação Brasileira de Criadores de Zebu (ABCZ), criados no Nordeste do Brasil. As características estudadas foram os pesos ajustados aos 205 (P205), 365 (P365) e 550 (P550) dias de idades. Foram utilizadas informações de pedigree de bovinos criados em regime de pastagem nascidos no período de 1975 a 2007 e dados dos pesos ajustados aos 205 (P205), 365 (P365) e 550 (P550) dias de idade. Foram utilizados registros de um total de 14.342, 10.033 e 7.824 animais na matriz de parentesco para P205, P365 e P550, respectivamente.

Para a edição do banco de dados, análise descritiva, remoção dos erros provenientes de escrituração zootécnica, erros de medição na coleta dos dados e digitação de dados incorretos foi utilizado o programa Statistical analysis Software (SAS, 2003).

Para a escolha do modelo de melhor ajuste foi adotado o critério do Fator de Bayes (FB). Os valores do Fator de Bayes (FB) foram obtidos pelo valor do logaritmo da função de verossimilhança ( $-2 \log$ L), através do programa POSTGIBBSF90, após a geração das distribuições a posteriori.

Além dos efeitos fixos (sexo, ano, época de nascimento, regime alimentar, condição de criação e fazenda), foram incluídos nos modelos os seguintes efeitos:

Modelo animal 1 (MA1): efeito genético aditivo direto e materno, efeito de ambiente permanente e residual (Modelo completo);

Modelo animal 2 (MA2): efeito genético aditivo direto e materno e residual;

Modelo animal 3 (MA3): efeito genético aditivo direto e residual.

Os modelos propostos são representados, matricialmente, das seguintes formas:

$$
\begin{aligned}
& \text { MA1: } \\
& y=X \beta+Z a+M n+W e p+e \\
& \text { MA2: } y=\mathrm{X \beta}+\mathrm{Za}+\mathrm{Mn}+\mathrm{e} \\
& \text { MA3: } y=\mathrm{X \beta}+\mathrm{Za}+\mathrm{e}
\end{aligned}
$$

Em que:

$\mathrm{y}=$ vetor de observações da característica avaliada (P205, P365 e P550);

$\beta=$ vetor de efeitos fixos de grupo contemporâneo no modelo, associados a y através da matriz de incidência de X;

$\mathrm{a}=$ vetor dos efeitos aleatórios de valor genético aditivo direto do animal, associados a y através da matriz de incidência $Z$;

$\mathrm{m}=$ vetor dos efeitos aleatórios de valor genético aditivo materno, associados a y através da matriz de incidência $\mathrm{M}$;

ep $=$ vetor dos efeitos aleatórios de ambiente permanente da vaca, associados a y através da matriz de incidência $\mathrm{W}$; 
$\mathrm{e}=$ vetor dos efeitos residuais

Os componentes de covariâncias e predição dos parâmetros genéticos foram estimados pela abordagem bayesiana utilizando-se o programa GIBBS3F90 descrito por Misztal (2012).

$\mathrm{Na}$ implementação das análises, sob enfoque bayesiano, foi definido o número total de iterações, descarte (burn-in) e salvamento (thin) (Tabela 1). Para a estimação dos componentes de covariância uma priori não informativa foi assumida para todos os parâmetros analisados. A convergência da cadeia de Gibbs, os testes de Raftery e Lewis e de Geweke foram realizados pelo o pacote estatístico do software $\mathrm{R}$ ( $\mathrm{R}$ DEVELOPMENT CORE TEAM, 2008) Bayesiam Output Analysis - BOA (SMITH, 2007). Fora utilizado um nível de significância de 5\% para o teste Geweke sob a hipótese nula Ho. Neste caso, o teste considera Ho como convergência da cadeia, ou seja, não se deseja rejeitar Ho. Portanto, quanto maiores os valores de p-valor (acima de 0,05 ) mais próximos da convergência está a cadeia (GEWEKE,1992).

Tabela 1. Definição do número total de iterações, descarte e salvamento para as características de crescimento após teste de convergência.

\begin{tabular}{ccccc}
\hline Características (dias) & Cadeias & Burn-in & Thin & Total de amostras \\
\hline P205 & 330.000 & 30.000 & 15 & 20.000 \\
P365 & 220.000 & 20.000 & 11 & 18.182 \\
P550 & 550.000 & 50.000 & 22 & 22.728 \\
\hline
\end{tabular}

Para a formação dos grupos de contemporâneos (GC) foram consideradas as seguintes fontes de variação: sexo; ano; época de nascimento; regime alimentar condição de criação; e fazenda.

As estimativas das tendências genéticas, maternas e fenotípicas para as características avaliadas foram obtidas pela regressão linear ponderada da média da variável dependente (valores genéticos e pesos observados) sobre o ano de nascimento do animal, obtidas pelo procedimento PROC REG do programa Statistical analysis Software (SAS, 2003) a partir dos arquivos de saída do POSTGIBBS_SAMPLES/POSTGIBBSF90 (GIBBS3F90).

As correlações genéticas, fenotípicas e de ambiente foram obtidas por meio das análises bicaracterísticas, através da abordagem bayesiana utilizando-se o programa GIBBS3F90.

\section{RESULTADOS E DISCUSSÃO}

As médias dos pesos dos animais da raça Tabapuã (Tabela 2) para as três características de crescimento P205, P365 e P550 estão acima das médias observadas por Ribeiro et al. (2007) e Caires et al. (2012) em estudo com animais da raça Tabapuã no Nordeste do Brasil. A variação entre as médias de pesos em uma mesma raça pode ser resultante da variação genética, manejo alimentar, reprodutivo e sanitário adotado em cada região e fazenda, bem como dos programas de seleção e melhoramento genético adotado. $\mathrm{O}$ estudo atual e o de Caires et al. (2012) utilizaram o mesmo conjunto de dados, cujas maiores médias podem indicar melhorias no manejo geral, bem como melhorias genéticas, dado que o banco de dados é atualizado.

Tabela 2. Estatísticas descritivas para os pesos ajustados aos 205 (P205), 365 (P365) e 550 (P550) dias de idade em bovinos da raça Tabapuã do Nordeste do Brasil.

\begin{tabular}{ccccccc}
\hline Características (dias) & $\mathrm{N}^{\mathrm{o}}$ de observações & Média & DP & CV\% & Mínimo & Máximo \\
\hline P205 & 14342 & 175,71 & 30,59 & 17,43 & 86 & 274 \\
P365 & 10033 & 241,64 & 48,86 & 20,22 & 127 & 438 \\
P550 & 7824 & 320,93 & 69,90 & 21,78 & 166 & 569 \\
\hline
\end{tabular}

DP= Desvio Padrão; CV\% = Coeficiente de Variação

O modelo é uma representação matemática da medida de uma característica qualquer e, por definição, jamais é perfeito (BARBOSA et al. 2008). A comparação entre modelos serve para escolher o mais apropriado a ser utilizado nas análises. A escolha do modelo que melhor se ajustou aos dados utilizou o Fator de Bayes (FB), cujos menores valores para o FB são os mais indicados, uma vez que sugerem melhor ajustamento dos modelos (CARDOSO,
2008).

Foram observados menores valores do Fator de Bayes para P205 e P365 quando considerou-se o MA1, modelo animal completo, no qual foram levados em consideração os efeitos maternos e de ambiente permanente. Para a característica P550, o menor valor de FB foi observado quando considerou-se o MA2, modelo animal que inclui o efeito genético aditivo direto e materno e o residual (Tabela 3 ). 
A. P. OLIVEIRA et al.

Tabela 3. Valores de FB - Fator de Bayes para os pesos P205, P365 e P550 dias de idade de animais da raça Tabapuã do Nordeste do Brasil.

\begin{tabular}{cccc}
\hline \multicolumn{4}{c}{ Fator de Bayes (FB) } \\
\hline & \multicolumn{2}{c}{ Características } & P550 \\
\cline { 2 - 4 } Modelos & P205 & P365 & $79.800,40$ \\
MA1 & $125.305,16$ & $95.755,02$ & $79.758,94$ \\
MA2 & $125.589,06$ & $95.908,58$ & $79.810,08$ \\
\hline
\end{tabular}

MA1 = modelo animal completo; MA2= modelo animal sem efeito de ambiente permanente; e MA3= modelo animal apenas com efeito genético aditivo.

Baldi et al. (2010) verificaram a significância dos efeitos aleatórios com base no teste de razão de verossimilhança em cinco diferentes modelos e concluíram que houve redução significativa do FB quando os efeitos aleatórios aditivo materno e de ambiente permanente materno foram incluídos no modelo na raça Canchim.

$\mathrm{O}$ ajuste dos dados em relação ao critério FB permite uma avaliação genética mais precisa sem necessariamente analisar os componentes de variância, os quais por muitas vezes podem ser superestimados. Isso foi observado quando os efeitos maternos não foram incluídos no modelo e todos os componentes de variância e parâmetros genéticos se apresentaram superestimados. (Tabela 4).

A não inclusão do efeito de ambiente permanente no modelo MA2 superestimou a variância genética aditiva materna, quais sejam, $\hat{\sigma}^{2} \mathrm{~m}=122,21$ (P205),

$\hat{\sigma}^{2} \mathrm{~m}=262,47(\mathrm{P} 365), \hat{\sigma}^{2} \mathrm{~m}=296,16(\mathrm{P} 550)$ e, consequentemente, os valores da herdabilidade materna, sendo eles $h^{2} \mathrm{~m}=0,21$ (P205), $h^{2} \mathrm{~m}=0,20$ (P365) e $\mathrm{h}^{2} \mathrm{~m}=$ 0,14 (P550), (Tabela 4). Isso indica que ao não se incluir o efeito de ambiente permanente materno na análise parte da variância do efeito permanente inflaciona a variância materna.

Para o modelo que não incluiu a variância genética aditiva materna e a variância do efeito de ambiente permanente MA3 ficou evidenciado o valor superestimado da variância genética aditiva, fato que influenciou o valor da herdabilidade direta: $h^{2} d=0,43$ (P205); $\mathrm{h}^{2} \mathrm{~d}=0,39 \quad(\mathrm{P} 365) ; \mathrm{e} \mathrm{h}^{2} \mathrm{~d}=0,33 \quad$ (P550) (Tabela 4). Este resultado indica que quando não se inclui os efeitos maternos nas análises parte da variância materna é estimada como variância genética aditiva. O MA3 também forneceu os menores valores de variância fenotípica entre todos os modelos empregados. Caso seja utilizado na prática ocasionaria valores de parâmetros genéticos elevados e viciados. Esse modelo apresentou as maiores diferenças nos valores dos parâmetros genéticos quando comparados aos demais modelos.

As cova- (ôam) riâncias entre o efeito genético aditivo direto e materno foram negativas para as três pesagens nos três modelos comparados (Tabela 4). Bolignon et al. (2012) concluíram que problemas na estruturação de dados e insuficiência do pedigree geram estimativas tendenciosas para os componentes de covariância, principalmente valores negativos para a covariância entre os efeitos genéticos diretos e maternos e consequentemente para o parâmetro genético que correlaciona estes dois efeitos (rGam).

Fernandes e Ferreira (2000) estudaram sete diferentes modelos em animais da raça Charolês e concluíram que quando não se incluiu o efeito materno e de ambiente permanente nos modelos os mesmos não mostraram diferenças significativas a $1 \%$, quando comparados entre si, para a característica ganho de peso do nascimento à desmama.

Scarpati e Lôbo (1999) estudaram os aspectos genético-quantitativos do peso ao nascer $(\mathrm{PN})$ de animais da raça Nelore considerando simultaneamente os efeitos maternos de origem genética e de ambiente. Não encontraram diferença significativa ao compará-lo com o modelo em que apenas o efeito genético materno foi considerado. Nesse caso, o modelo com a ausência do efeito permanente foi considerado o mais adequado.

Cyrillo et al. (2004) e Jacinto et al. (2005) não recomendam a escolha do modelo que contempla somente o efeito genético aditivo direto, pois na maioria dos casos este apresenta diferenças significativas dos demais modelos que incluem os efeitos maternos, bem como a superestimação dos componentes de covariância e parâmetros genéticos.

Dias et al. (2005) afirmam que a não inclusão dos efeitos maternos no modelo de análise pode fazer com que as variâncias do efeito permanente e genética aditiva materna inflacionem a variância genética aditiva direta. 
A. P. OLIVEIRA et al.

Tabela 4. Estimativas dos componentes de covariância e parâmetros genéticos dos pesos ajustados aos 205 (P205), 365 (P365) e 550 (P550) dias de idade obtidas em análise de característica única para os diferentes tipos de modelos estatísticos em bovinos da raça Tabapuã do Nordeste do Brasil.

\begin{tabular}{|c|c|c|c|c|c|c|c|c|c|}
\hline \multirow{2}{*}{ Modelos } & \multicolumn{9}{|c|}{ P205 } \\
\hline & $\hat{\sigma}_{\mathrm{a}}^{2}$ & $\hat{\sigma}_{\mathrm{m}}^{2}$ & $\hat{\sigma}_{\mathrm{am}}$ & $\hat{\sigma}_{\text {ep }}^{2}$ & $\hat{\sigma}_{\mathrm{e}}^{2}$ & $\hat{\sigma}_{\mathrm{p}}^{2}$ & $\mathrm{~h}_{\mathrm{d}}^{2}$ & $\mathrm{~h}_{\mathrm{m}}^{2}$ & $\mathrm{rG}_{\mathrm{am}}$ \\
\hline MA1 & 108,20 & 79,53 & $-30,88$ & 38,40 & 324,36 & 550,49 & 0,20 & 0,14 & $-0,32$ \\
\hline MA2 & 104,22 & 122,01 & $-38,05$ & --- & 332,54 & 558,76 & 0,19 & 0,21 & $-0,33$ \\
\hline \multirow[t]{3}{*}{ MA3 } & 235,69 & --- & --- & --- & 311,68 & 547,37 & 0,43 & --- & --- \\
\hline & \multicolumn{9}{|c|}{ P365 } \\
\hline & $\hat{\sigma}_{\mathrm{a}}^{2}$ & $\hat{\sigma}_{\mathrm{m}}^{2}$ & $\hat{\sigma}_{\mathrm{am}}$ & $\hat{\sigma}_{\mathrm{ep}}^{2}$ & $\hat{\sigma}_{\mathrm{e}}^{2}$ & $\hat{\sigma}_{\mathrm{p}}^{2}$ & $\mathrm{~h}_{\mathrm{d}}^{2}$ & $\mathrm{~h}_{\mathrm{m}}^{2}$ & $\mathrm{rG}_{\mathrm{am}}$ \\
\hline MA1 & 355,56 & 185,14 & $-120,46$ & 69,85 & 706,73 & 1317,28 & 0,27 & 0,14 & $-0,46$ \\
\hline MA2 & 352,46 & 262,47 & $-134,63$ & --- & 719,37 & 1334,30 & 0,26 & 0,20 & $-0,43$ \\
\hline \multirow[t]{3}{*}{ MA3 } & 478,43 & --- & --- & --- & 742,82 & 1221,25 & 0,39 & --- & --- \\
\hline & \multicolumn{9}{|c|}{ P550 } \\
\hline & $\hat{\sigma}_{\mathrm{a}}^{2}$ & $\hat{\sigma}_{\mathrm{m}}^{2}$ & $\hat{\sigma}_{\mathrm{am}}$ & $\hat{\sigma}_{\mathrm{ep}}^{2}$ & $\hat{\sigma}_{\mathrm{e}}^{2}$ & $\sigma_{p}^{2}$ & $\mathrm{~h}_{\mathrm{d}}^{2}$ & $\mathrm{~h}_{\mathrm{m}}^{2}$ & $\mathrm{rG}_{\mathrm{am}}$ \\
\hline MA1 & 457,34 & 222,87 & $-98,72$ & 79,78 & 1365,09 & 2125,09 & 0,21 & 0,10 & $-0,28$ \\
\hline MA2 & 455,08 & 296,16 & $-104,60$ & --- & 1382,60 & 2133,84 & 0,21 & 0,14 & $-0,26$ \\
\hline MA3 & 680,66 & --- & --- & --- & 1384,20 & 2064,86 & 0,33 & --- & --- \\
\hline
\end{tabular}

MA1 $=$ modelo animal completo; MA2 = modelo animal sem efeito permanente; e MA3 = modelo animal apenas com efeito genético aditivo.

$\sigma_{\mathrm{a}}^{2}=$ variância genética aditiva direta; $\sigma_{\mathrm{m}}^{2}=$ variância genética aditiva materna; $\sigma_{\text {ep }}^{2}=$ variância de ambiente permanente; $\sigma_{\mathrm{e}}^{2}=$ variância residual; $\sigma_{\mathrm{p}}^{2}=$ variância fenotípica; $\mathrm{h}_{\mathrm{d}}^{2}=$ herdabilidade direta; e $\mathrm{h}_{\mathrm{m}}^{2}=$ herdabilidade materna.

A seleção de animais com potencial genético para características de crescimento é frequentemente adotada dentro dos programas de melhoramento animal. Uma das maneiras de se avaliar o progresso genético, para cada característica, é através do cálculo da tendência genética da população (Tabela 5), quantificando quanto da variação anual do rebanho tem origem genética.

Tabela 5. Equações de regressão para os efeitos genético, materno e fenotípico para os pesos aos 205 (P205), 365 (P365) e 550 (P550) em bovinos da raça Tabapuã do Nordeste do Brasil.

\begin{tabular}{|c|c|c|c|}
\hline Características & Equação & $\mathrm{R}^{2}$ & p-valor \\
\hline \multicolumn{4}{|c|}{ Genético } \\
\hline P205 & $Y=-164,43+0,0834 *$ ano & 0,25 & $0,0031 * *$ \\
\hline P365 & $Y=-54,95+0,2296^{*}$ ano & 0,57 & $0,0001 * *$ \\
\hline P550 & $\mathrm{Y}=-515,58+0,2601 *$ ano & 0,62 & $0,0001 * *$ \\
\hline \multicolumn{4}{|c|}{ Materno } \\
\hline P205 & $Y=-4,4553+0,0073 *$ ano & 0,0038 & $0,7337^{\mathrm{ns}}$ \\
\hline \multicolumn{4}{|c|}{ Fenotípico } \\
\hline P205 & $\mathrm{Y}=-1497,36+0,8383 *$ ano & 0,56 & $0,0001 * *$ \\
\hline P365 & $Y=-159,91+0,7011 *$ ano & 0,22 & $0,0067 * *$ \\
\hline P550 & $\mathrm{Y}=582,412-0,1333 *$ ano & 0,0034 & $0,7495^{\mathrm{ns}}$ \\
\hline
\end{tabular}

$\mathrm{R}^{2}=$ coeficiente de determinação; **Significativo a $1 \%$; ${ }^{\text {ns }}$ Não significativo.

A tendência genética (regressão linear) para a característica P205, quando utilizado o modelo completo MA1, foi significativa $(\mathrm{p}<0,0001)$ e igual a $0,0834 \mathrm{~kg} / \mathrm{ano}$, equivalendo a 2,67 kg (Tabela 5) durante os 32 anos avaliados. Em termos de mudança genética anual, representa incremento de $0,05 \%$ na média do peso por ano.

Para a característica P365, quando utilizado o modelo completo MA1, a mesma apresentou tendência genética (regressão linear) de efeito direto significativa $(\mathrm{p}<0,0001)$ e igual a $0,2296 \mathrm{~kg} /$ ano (Tabela 5 ), equivalente a $7,35 \mathrm{~kg}$ durante os 32 anos avaliados. Em termos de mudança genética anual representa incremento de $0,09 \%$ por ano.

Para a característica P550, quando utilizado o modelo completo MA1, a mesma apresentou tendên- 
cia genética (regressão linear) de efeito direto significativa ( $<<0,0001$ ) e igual a $0,2601 \mathrm{~kg} /$ ano (Tabela 5 ), o que equivale a $8,32 \mathrm{~kg}$ durante os 32 anos avaliados. Em termos de mudança genética anual representa incremento de $0,08 \%$ por ano.

Os resultados obtidos neste trabalho, mesmo que em pequenas magnitudes, demonstram tendências genéticas positivas para as características estudadas, o que indica um progresso genético para as características de crescimento na raça Tabapuã da região Nordeste durante o período avaliado (32 anos).

As tendências genéticas para os efeitos maternos (regressão linear) não foram significativas $(\mathrm{p}>0,05)$ para todas as características.

A obtenção de valores genéticos depende de métodos estatísticos sofisticados, capazes de indicar quais animais devem ser selecionados a partir da estrutura de dados disponíveis (CARNEIRO JÚNIOR et al., 2010).

$\mathrm{O}$ estudo das tendências fenotípicas tem como principal objetivo monitorar o progresso fenotípico, avaliar conjuntamente a atuação dos programas de seleção e a melhoria das condições ambientais sobre as características analisadas ao longo dos anos. A tendência fenotípica (regressão linear) para o peso aos 205 dias de idade (P205) foi significativa $(\mathrm{P}<0,0001$ ) e igual a $0,8383 \mathrm{~kg} /$ ano (Tabela 5$)$, o que corresponde a um incremento de $26,82 \mathrm{~kg}$ na característica durante o período analisado. Em termos de mudança fenotípica anual, esse valor representa para P205 ganhos de $0,48 \%$.

Malhado et al. (2008), estudando animais da raça Nelore do estado da Bahia no período de 1955 a
2007, verificaram valores para a tendência fenotípica das características P205, P365 e P550 iguais a 1,40, 1,65 e $2,11 \mathrm{~kg} / \mathrm{ano}$, respectivamente.

Os resultados para a tendência fenotípica com poucos ganhos e baixos valores de $\mathrm{R}^{2}$ evidenciam baixo progresso durante o período estudado no presente trabalho e grande variação nos valores genéticos dos touros nos períodos avaliados. Esse fato é decorrente das poucas melhorias das condições de criação no rebanho.

A tendência fenotípica (regressão linear) para o peso aos 365 dias de idade (P365) foi significativa $(\mathrm{P}<0,001)$ e igual a $0,7011 \mathrm{~kg} /$ ano (Tabela 5), correspondendo a um incremento de $22,42 \mathrm{~kg}$ na característica durante o período analisado. Em termos de mudança fenotípica anual, esse valor representa para P365 ganhos de 0,29\%. Houve ganho genético para as características P205 e P365. Entretanto, o progresso observado neste estudo deve ser atribuído principalmente ao ambiente, visto que a contribuição genética, apesar de positiva, foi baixa.

Para a característica P550 a tendência fenotípica (regressão linear) não foi significativa $(p>0,05)$ (Tabela 5).

Em análises multicaracterísticas a avaliação de um animal para uma característica se faz pela contribuição de todas as outras características envolvidas. Através dessas análises é possível obter as correlações genéticas, fenotípicas e de ambiente.

As correlações genéticas entre o peso aos 205 , 365 e 550 dias de idade foram de alta magnitude $(0,90 ; 0,90$ e 0,98$)$, mostrando que a maioria dos genes que atuam no peso aos 205 dias também atuam aos 365 e 550 dias de idade (Tabela 6).

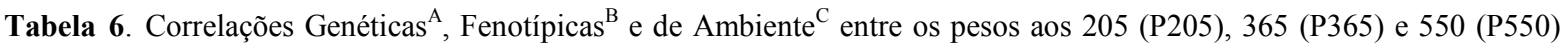
dias de idade em bovinos da raça Tabapuã do Nordeste do Brasil.

\begin{tabular}{cccc}
\hline \multirow{2}{*}{ Características } & \multicolumn{3}{c}{ Características } \\
\cline { 2 - 4 } & P205 & P365 & P550 \\
\hline P205 & - & 0,90 & 0,90 \\
P365 & 0,71 & - & 08 \\
& $\mathbf{0 , 1 9}$ & 0,88 & \\
P550 & 0,51 & $\mathbf{0 , 1 0}$ & - \\
\hline
\end{tabular}

${ }^{\mathrm{A}}$ Acima da diagonal; ${ }^{\mathrm{B}}$ Abaixo da diagonal, primeiro valor; $\mathrm{e}^{\mathrm{C}}$ Abaixo da diagonal (negrito).

Apesar da correlação entre P365 e P550 ter sido muito alta $(0,98)$, selecionar animais aos 205 dias de idade (P205) seria muito razoável devido a possibilidade de ganhos genéticos via resposta correlacionada e possibilitar a redução do intervalo de geração. Santos et al. (2005) encontraram para $\mathrm{P} 205 \mathrm{P} 365=0,90, \mathrm{P} 205 \mathrm{P} 365=0,81$ e $\mathrm{P} 365 \mathrm{P} 550=0$, 97 em bovinos da raça Nelore Mocha. Cucco et al. (2010), em animais da raça Pardo Suíço, encontraram para $\mathrm{P} 205 \mathrm{P} 365=0,91, \mathrm{P} 205 \mathrm{P} 550=0,71 \mathrm{e}$ $\mathrm{P} 365 \mathrm{P} 550=0,91$. De modo geral, são esperadas correlações altas entre as pesagens, pois o peso a idade mais jovem é um componente do peso a idade posterior.
As correlações genéticas entre P205 e P365, P205 e P550, e P365 e P550 foram superiores em magnitude à fenotípica $(0,71 ; 0,51$ e 0,88$)$ (Tabela 6). Embora pareça improvável, pois a fenotípica contém a genética, Searle (1961) demonstrou que as correlações fenotípicas podem ser menores que as genéticas, desde que associadas a correlações ambientais baixas e positivas, quando os genes que controlam duas características quaisquer são similares. As correlações ambientais entre o peso aos 205, 365 e 550 dias de idade $(0,19,0,39$ e 0,10$)$ (Tabela 6) foram de baixa magnitude. 


\section{CONCLUSÕES}

$\mathrm{O}$ modelo que inclui o efeito materno e de ambiente permanente (MA1) proporciona melhor ajuste aos dados para as características P205 e P365. Para a característica P550 o modelo que proporciona o melhor ajuste é aquele que inclui o efeito genético aditivo direto e materno e o residual (MA2).

Quando não se considera um ou mais efeitos (materno e/ou de ambiente permanente) no modelo tem-se diferenças que poderão alterar as estimativas dos parâmetros genéticos.

Observa-se pequeno progresso genético no período entre 1975 a 2007 para as características P205, P365 e P550.

As características de crescimento analisadas apresentam variabilidades genéticas aditivas para que a resposta à seleção seja positiva.

É possível obter ganhos genéticos, via resposta correlacionada, para os pesos aos 365 e 550 dias de idade ao selecionar para P205.

\section{AGRADECIMENTOS}

À UESB, FAPESB e CNPq pelo apoio financeiro e à $\mathrm{ABCZ}$, pela cessão dos dados.

\section{REFERÊNCIAS}

ASSOCIAÇÃO BRASILEIRA DOS CRIADOS DE TABAPUÃ. Disponível em: <http:// tabapua.org.br/ raca-tabapua/historia-da-raca/>. História da Raça . Acessado em 11 de Maio de 2014.

BALDI, F.; ALENCAR, M. M.; ALBUQUERQUE, L. G. Estimativas de parâmetros genéticos para características de crescimento em bovinos da raça Canchim utilizando modelos de dimensão finita. Revista Brasileira de Zootecnia, Viçosa, v. 39, n. 11, p. 2409-2417, 2010.

BARBOSA, L. et al. Estimação de parâmetros genéticos em suínos usando Amostrador de Gibbs. Revista Brasileira de Zootecnia, Viçosa, v. 37, n. 7, p. 1200-1206, 2008.

BOLIGON, A. A. et al. Influence of dat struture on the estimation of the additive genetic direct and maternal covariance for early growth traits in Nellore cattle. Livestock Science, Amsterdam: Elsevier B.V., v. 145, n.1, p. 212-218, 2012.

CAIRES, D. N. et al. Tabapuã breed in Northeastern Brazil: genetic progress and population structure. Revista Brasileira de Zootecnia, Viçosa, v. 41, n. 8, p. 1858-1865, 2012.
CARDOSO, F. F. Manual de utilização do Programa INTERGEN - Versão 1.0 em estudos de genética quantitativa animal. Bagé. Embrapa Pecuária Sul, 2008. 75p. ( Documentos / Embrapa Pecuária Sul, ISSN 1982-5390; 74).

CARNEIRO JÚNIOR, J. M. et al. Predição de valores genéticos utilizando inferência bayesiana e frequentista em dados simulados. Acta Scientiarum Animal Sciences, Maringá, v. 32, n. 3, p. 337-344, 2010.

CUCCO, D. C. et al. Genetic parameters for postweaning traits in Braunvieh cattle. Genetics and Molecular Research, Ribeirão Preto, v. 9, n.1, p. 545-553, 2010.

CYRILLO, J. N. S.G. et al. Modelagem e estimação de parâmetros genéticos e fenotípicos para pesos do nascimento à seleção (378 dias) de machos Nelore. Revista Brasileira de Zootecnia, Viçosa, v. 33, n. 6, p. 1405-1415, 2004.

DIAS, L.T. et al. Estimação de Parâmetros Genéticos para Peso em Diferentes Idades para Animais da Raça Tabapuã. Revista Brasileira de Zootecnia, Viçosa, v. 34, n. 6, p. 1914-1919, 2005.

FERNANDES, H. D.; FERREIRA, G. B. Estudo comparativo de sete modelos estatísticos para a característica ganho de peso em bovinos de corte. Revista Brasileira de Zootecnia, Viçosa, v. 29, n. 5, p. 1340-1348, 2000.

GEWEKE, J. Evaluating the accuracy of samplingbased approaches to the calculation of posterior moments. In: BERNARDO, J.M. BERGER, J.O., DAWID, A.P. \& SMITH, A.F.M. (Ed.) Bayesian Statistics 4. New York: Oxford University, 1992. cap.4, p.169-194.

JACINTO, E. J. et al. Adequação de modelos para estimativa de parâmetros genéticos relativos ao peso aos P205 de idade em bovinos da raça Tabapuã, criados na região Nordeste. Revista Ciência Agronômica, Fortaleza, v. 36, n. 2, p. 221-226, 2005.

MALHADO, C. H. M. et al. Progresso genético e estrutura populacional do rebanho Nelore no Estado da Bahia. Pesquisa Agropecuária Brasileira, Brasília, v. 43, n. 9, p. 1163-1169, 2008.

MISZTAL,J.2012. Fortran Programs. Disponível em: $<$ http://nce.ads.uga.edu/wiki/doku.php?id= Readme.gibbs3>. Acesso em 13 de outubro 2012.

RAFTERY, A. L.; LEWIS, S. One long run with diagnostics: implementation strategies 474 for Markov chain Monte Carlo [Comment]. Statistical Science, Hayward, v. 7, n. 4, p. 475 493-497, 1992. 
R DEVELOPMENT CORE TEAM. R: a language and environment for statistical computing. $R$ Foundation for Statistical Computing, Vienna, Austria ,2008. Disponível em: <http://www.r-project.org/ >. Acesso em outubro de 2012.

RIBEIRO, S. H. A. et al. Estudo genéticoquantitativo de características de crescimento na raça Tabapuã. Arquivo Brasileiro de Medicina Veterinária e Zootecnia, Belo Horizonte, v. 59, n. 2, p. 473-480, 2007.

SANTOS, P. F. et al. Correlação genética, fenotípica e ambiental do desenvolvimento ponderal em bovinos da raça nelore variedade mocha. Archives of Veterinary Science, Curitiba, v. 10, n. 2, p. 55-60, 2005.

SAS INSTITUTE. Statistical Analysis System user's guide. Version 9.1. Cary, USA, 2003.

SCARPATI, M. T. V.; LÔBO, R. B. Modelos animais alternativos para estimação de componentes de covariância e de parâmetros genéticos e fenotípicos de peso ao nasce na raça Nelore. Revista Brasileira de Zootecnia, Viçosa, v. 28, n. 3,p. 512-518,1999.

SEARLE, S.R. Variance components in the unbalanced 2-way nested classification. Annals of Mathematical Statistics, Durham, North Carolina, v. 32, n.4, p. 1161-1166, 1961.

SMITH, B. J. Bayesian output analysis program (BOA) version 1.1.7.2 User's manual. Iowa: University Of Iowa., 2007. 